\title{
Impact of the face angle to traveling trajectory during the riding standing-type personal mobility device
}

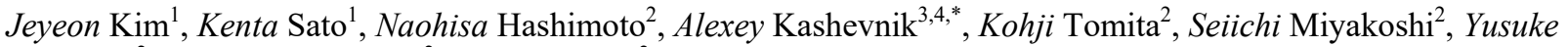 \\ Takinami $^{2}$, Osamu Matsumoto ${ }^{2}$ and Ali Boyali $^{2}$ \\ ${ }^{1}$ Department of Creative Engineering, National institute of Technology, Tsuruoka College, 997-8511 Tsuruoka Yamagata, Japan \\ ${ }^{2}$ Intelligent System Research Institute, National institute of Advanced Industrial Science and Technology, 305-8560 Tsukuba Ibaraki, \\ Japan \\ ${ }^{3}$ SPIIRAS, 39, 14th Line, St. Petersburg 199178, Russia \\ ${ }^{4}$ ITMO University, 49 Kronverksky Pr, St. Petersburg, Russia
}

\begin{abstract}
In this paper, we investigate the impact of face direction during traveling by Standing-Type Personal Mobility Device (PMD). The use of PMD devices has been a popular choice for recreational activities in the developed countries such as in the USA and the countries in Europe. These devices are not completely risk free and various accidents have been reported. Since that, the risk factors leading to accidents have to be investigated. Unfortunately, the research studies on the risk factors on riding PMD devices have not been matured as much as the studies on driving cars. In this paper, we evaluate the impacts of face angle on travelling trajectory during travelling in a PMD. We showed by experiments that, the face direction is an important factor in risk assessment for traveling by a PMD.
\end{abstract}

\section{Introduction}

In the recent years, researches on personal mobility devices (PMD) have been actively conducted, as these devices are promising alternatives in solving the first and last mile. The PMDs can be classified into standing type PMDs, two-seater ultra-small devices and electric bicycles. Among them, the standing type PMDs have gained a great deal reputation due to their unique motion capabilities. However, the standing type PMDs have not been allowed in the public places due to the lack of regulations, insufficient research studies for assessment of their safety for policy makers. In Japan, traveling by a PMD is prohibited as well.

To enable the research on PMDs and their interaction with pedestrian and other entities in the public places, Japan has designated a special zone in which the PMDs are allowed. Various studies such as social acceptability, attitude towards them, safety, usability evaluation of the standing-type PMD have been published [1,2]. Despite the fact that there have been frequent accidents caused by rider's behaviors reported due to the trends in increasing use of PMDs in the USA, and Europe [3, 4], inattentive operation and distracted ride profiles [5] have been less studied in the literature up to date.

Distracted driving is any activity that diverts attention from driving, including talking or texting on smartphone, eating, drinking, talking to other people, fiddling with the vehicle infotainment or navigation system [5]. That is, it indicates a driving operation in a state in which the rider does not concentrate on the operation of the device or concentrate on other activities than the required for the ride. From the research [8], it was found that the safety education contributes to reduce distracted driving [8]. Various studies have been also made on stick driving, dozing driving, etc. to prevent distracted driving.

The rest of the paper is organized as follows. Section 2 describes the mobile application developed for rider face tracking. Section 3 presents experiments methodology. Section 4 describes the results of the experiments. Finally, the conclusion summarize the paper.

\section{Related work}

The section presents research and development in the area of mobile applications utilisation for riders and drivers assistance.

The paper [9] describes the CarSafe driver safety application for Android phones that detects and alerts drivers to dangerous driving conditions and behaviour. It uses computer vision and machine learning algorithms on the phone to monitor and detect whether the driver is tired or distracted using the front-facing camera, while at the same time tracking road conditions using the rearfacing camera. CarSafe also tries to solve the problem of processing video streams from both the front and rear cameras simultaneously by using a context-aware algorithm. This app has two main disadvantages. The first problem is a lack of emotions, gestures and speech recognition. The second one is that it is not available in any application store.

* Corresponding author: alexey@iias.spb.su 
The paper [10] presents a DriveSafe driver safety application for iPhones that detects inattentive driving behaviors and gives corresponding feedback to drivers, scoring their driving and alerting them in case their behaviors are unsafe. It uses computer vision and pattern recognition techniques on the iPhone to assess whether the driver is drowsy or distracted using the rear-camera, the microphone, the inertial sensors and the GPS.

The paper [11] describes WalkSafe application for Android-based smartphone that aids people that walk and talk, improving the safety of pedestrian mobile phone users. WalkSafe uses the back camera of the mobile phone to detect vehicles approaching the user, alerting the user of a potentially unsafe situation; more specifically This application uses machine learning algorithms implemented on the phone to detect the front views and back views of moving vehicles and exploits phone APIs to save energy by running the vehicle detection algorithm only during active calls. WalkSafe alerts the user of unsafe conditions using sound and vibration from the phone.

The paper [12] provides comparative analysis of the behaviors of non-professional and professional drivers on the basis of the undertaken individual studies. The results showed the correlation between non-professional drivers and ordinary and aggressive violations and errors, while professional drivers were associated with positive behaviors.

Authors of the paper [13] examines older people driving behavior which includes road selection, left/right turn and driving speed. There are 108 participants has been participated in the study. Authors confirmed that older drivers are more reluctant to drive on expressway than other participants for long trips. Considering that driving in non-expressway with long time may cause frequently facing complex and unsafe conditions, more efforts should be carried out to promote and train expressway driving among older drivers.

The paper [14] describes a tracking system that monitors facial feature and create $3 \mathrm{D}$ geometric reasoning. The system has three main components: robust facial feature tracking, head pose and gaze estimation, and 3D geometric reasoning to detect Eyes Off the Road state.

The system proposed authors in [15] is the one that determines dangerous states by sound. Authors present silence removal approach using short term energy and zero crossing rate prior to extracting the relevant features in order to reduce the computational time in a vehicular environment.

\section{Rider face tracking}

Modern smartphones are equipped with front-facing camera and variety of built-in sensors such as an accelerometer, a gyroscope, an ambient light sensor, a proximity sensor, a magnetic field sensor, and GPS, that are capable to measure parameters that are needed determine dangerous states.

The authors proposed the rider assistance system using a personal smartphone for PMDs [16]. The evaluation of this application was reported in [17] using a traveling simulator. In the paper [16] the reference model of a two-wheeled self-balancing vehicles rider assistant system is presented. The model consists of five main modules: mobile application, cameras, sensors, local database and cloud service. These modules allow the system to recognize rider and vehicle behaviour and producing alerts and warnings when dangerous situations are detected. The authors also detailed appropriate ontologies for the rider and vehicle behaviour recognized as well as the Segway motion detection algorithms using an Android-based smartphone. The cloud service is used for keeping statistics of the system utilisation.

At the moment the rider assistance system determines two dangerous states: drowsiness and distraction. The smartphone's front-facing camera monitors the head movements, facial expressions, and the prolonged and frequent eye blinks that indicates the micro sleep. Visual cues relevant to the drowsiness state are percentage of closure of eyelid (PERCLOS), eye blink time, eyeblinking rate, eye gaze, pupil movement and eyelid movement. Distraction dangerous state is related to maintaining the eye contact with the road. Distraction occurs when drivers divert their attention away from the driving task to focus on another activity instead.

Using the smartphone the rider assistant system generates alerts for the driver using vibration, audible signals, and visual information in according with the proposed recommendation schemes. The rider assistant system provides statistics about the driver's behaviour on his/her route. It tracks such parameters as recognized dangerous states, time, location, speed, acceleration, driver's face parameters, and other information from smartphone sensors. During the experiments presented in the paper the face angle has been measured and saved in the cloud service. Table 1 shows the example statistics for distraction dangerous state. The statistics is stored in the cloud systems that provides possibility to use the powerful resources to analyses it. The use cases for rider assistant mobile application evaluation using a ride simulator is introduced in reference [17]. The ride simulator has been proposed and briefly explained the concept, system configuration, employed mobility, and preliminary experiments.

In these studies, the impact of the face direction angle on ride trajectory during a ride has not been taken into account, therefore, we contribute these studies by investigating the effects of face direction angle based on the developed by authors [16] mobile application that has been adapted to us for PMD. Although there are rider assistance systems being studied, there has been no risk assessment from rider's perspective for PMD use. In this paper, we examine the risks associated by the riders by analysing the influence of face direction using a smartphone application. 
Table 1. Statistics example accessible after the rider assistance system.

\begin{tabular}{|c|c|c|c|c|c|c|c|}
\hline Date/Time & Latitude & Longitude & $\begin{array}{c}\text { Acceleration } \\
\mathbf{( m / \mathbf { s } ^ { \wedge } \mathbf { 2 } )}\end{array}$ & $\begin{array}{c}\text { Head } \\
\mathbf{a n g l e}\end{array}$ & $\begin{array}{c}\text { Speed } \\
\mathbf{( k m / h )}\end{array}$ & City/Country & $\begin{array}{c}\text { Light level } \\
(\mathbf{L u x})\end{array}$ \\
\hline $\begin{array}{c}27 / 04 / 17 \\
18: 38: 08: 048\end{array}$ & 59,94228254 & 30,262599 & 11,16 & $-22,51$ & 12 & City & 116 \\
\hline $\begin{array}{c}13 / 05 / 17 \\
16: 06: 32: 875\end{array}$ & 56,18414201 & 36,9833062 & 2,1 & 20,43 & 26 & City & 630 \\
\hline $\begin{array}{c}13 / 05 / 17 \\
16: 08: 35: 733\end{array}$ & 56,19890477 & 36,95948511 & 2,09 & $-26,25$ & 64 & City & 565 \\
\hline $\begin{array}{c}13 / 05 / 17 \\
16: 08: 54: 948\end{array}$ & 56,20137014 & 36,95550604 & 4,08 & 35,21 & 80 & Country & 465 \\
\hline $\begin{array}{c}13 / 05 / 17 \\
16: 36: 43: 879\end{array}$ & 56,38791051 & 36,66605464 & 9 & 32,82 & 67 & Country & 731 \\
\hline
\end{tabular}

\section{Methodology of experiments}

In the experiments a Segway PMD [11] and collected position information by Total Station range sensors by Lacia Geosystems (TM, R) is used. The face direction angle of the subjects during the rides using the smartphone application presented in [9] is recorded. An Android-based smartphone was attached on the Segway as shown in Figure 1 and was synchronized with Total Station. The time synchronization error was measured around one second. The experiments were carried out in a gymnasium of Tsuruoka College to eliminate the effects of bumpy road and irregular road surfaces on the trajectories.

The experimental method is shown in Figure 2. The subjects gaze at the target and travel straight from the start point to the goal. Four subjects participated in the experiments who are between 19 and 22 years old. Each of the subjects repeated the experiment seven times with a speed of $4 \mathrm{mile} / \mathrm{h}$. The starting position is chosen as the origin in the experiments.

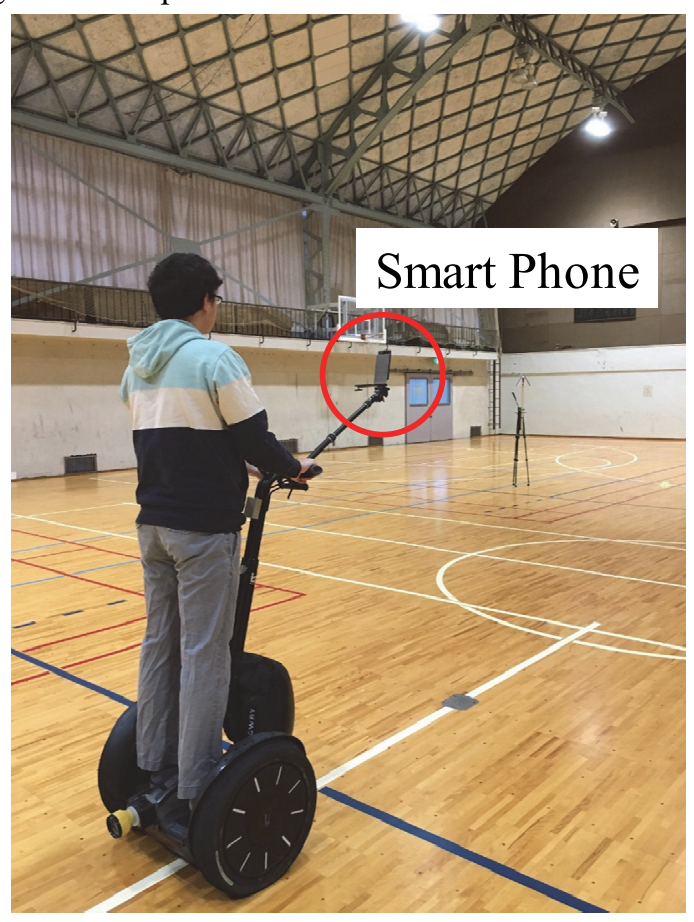

Fig. 1. Installation of Smartphone.

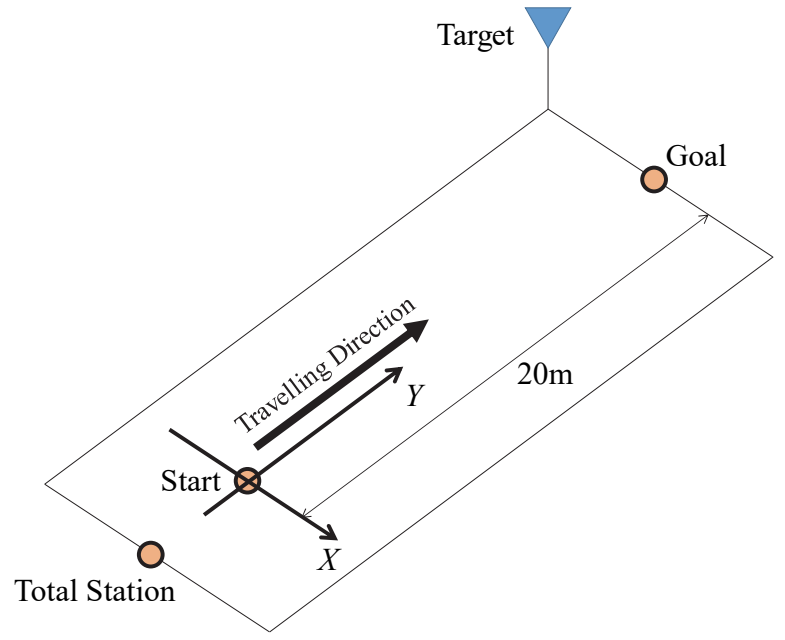

Fig. 2. Method of experiments.



Fig. 3. Scene of experiments. 


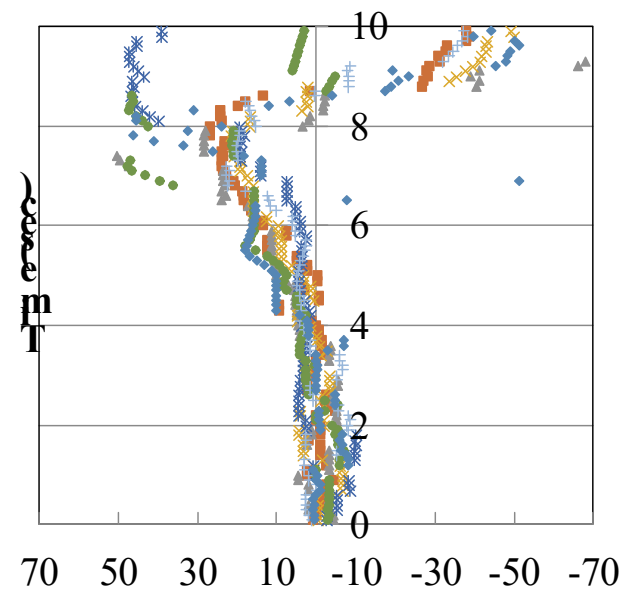

(a) Face angle

Angle(left:+)

Fig. 4. A result of travelling experiment (Subject 1).

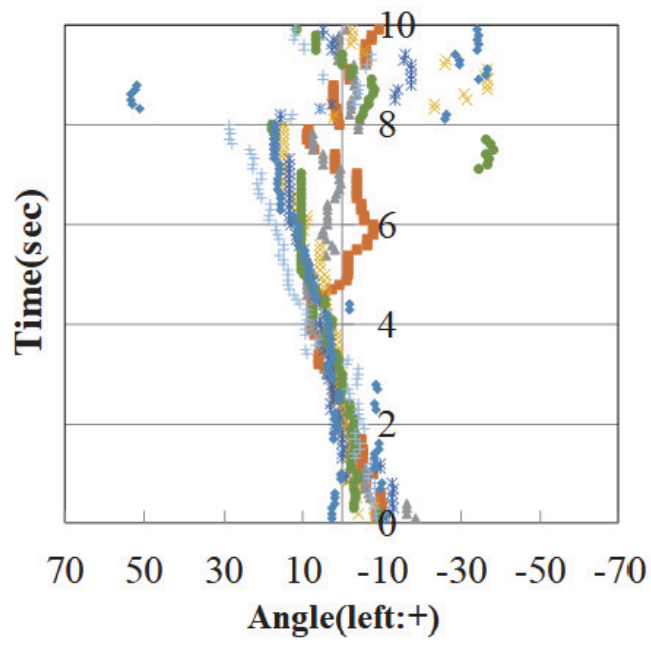

(a) $\mathrm{H}$

Fig. 5. A result of travelling experiment (Subject 4).

The subjects travel along the $\mathrm{y}$ axis. The $\mathrm{x}$-axis shows the lateral direction (Fig. 2.). The positive face direction angle (clockwise) is towards to the right side of the subjects.

\section{Results}

The inputs to the assessments are the face direction angle and trip trajectory. Figures 4 and 5 show the face angle and the traveling trajectory in the experiments. In Figure 4 (a), the horizontal axis is the face angle and the vertical axis is time. In Figure 4 (b), the horizontal axis is the position in the $\mathrm{x}$ direction and the vertical axis is the time.

As shown in Figure 4(a), it can be seen that the face angle increases as the user gazes while watching the target. Then, it shows that the deviation (error) in the $\mathrm{x}$ direction increases as the face angle increases (Fig. 4 (b)). That is, it can be seen that a deviation occurs in the opposite direction to the gaze direction. Three of the subjects showed this tendency. Figure 5 shows an example in which the deviation in the $\mathrm{x}$ direction is not
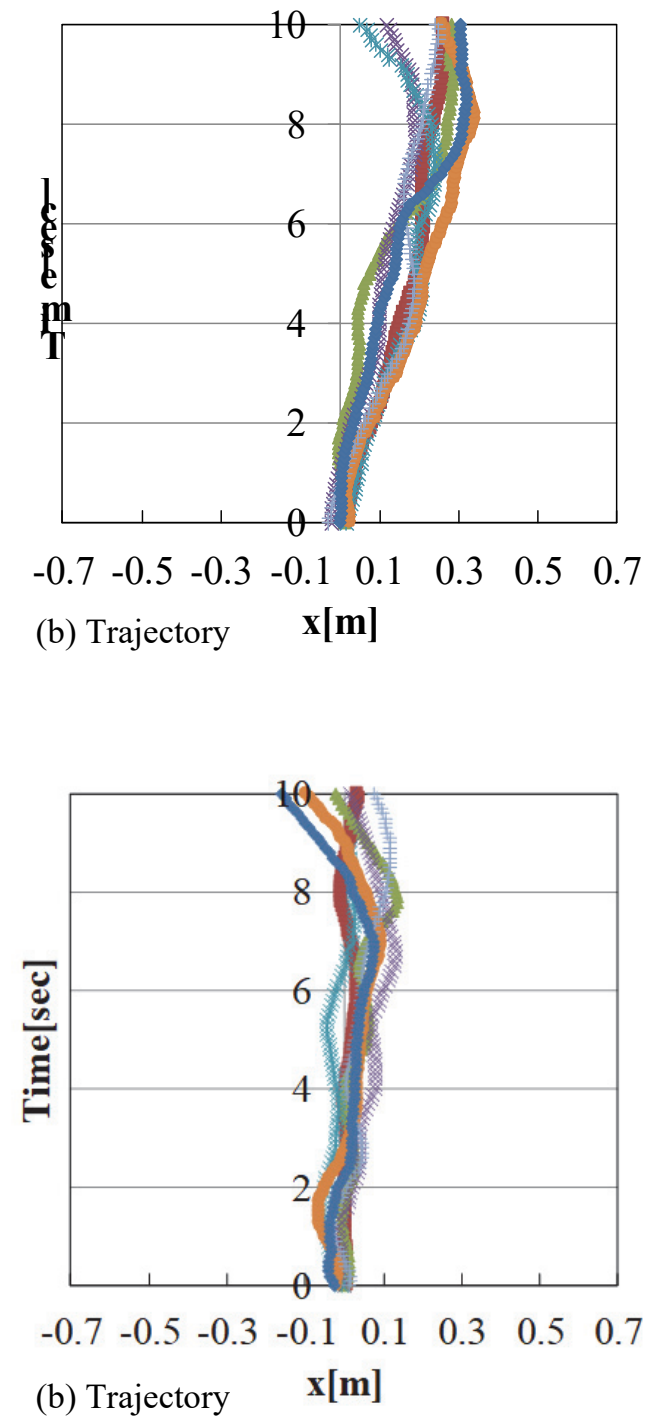

as much large as that of other participants while the face direction angle is increasing.

The experiments show that, there is a strong correlation between the face direction angle and the direction of the traveling trajectory. The deviation of the PMDs from the intended trajectory while the face direction changing create suspicion that the riders change the body center of gravity without realizing it while gazing at the target.

\section{Conclusions}

In this paper, we investigated the influence on the traveling trajectory by the angle of the rider face during travelling for risk assessment in PMD. Specifically, we conducted a running test in the indoor, and examined the relationship between the face angle during running and the traveling locus. As the experimental results show, the larger the face angle, the more the deviation shows that the deviation of the traveling locus becomes larger. In addition, it showed that the deviation of the running locus occurred in the direction opposite to the direction the subject was closely watching. These results showed

\footnotetext{
*Corresponding author: alexey@iias.spb.su
} 
how distracted driving is dangerous and causes the risks of undeliberate trajectory changing.

There are outdoor experiments as future tasks. Indoor experiments were carried out without the influence of the slope of the road surface in this paper, but it is necessary to evaluate the risk caused by distracted driving outdoors.

This work was supported by JSPS KAKENHI Grant Number 16K18057, project funded by grant \# 17-29-03284 of the Russian Foundation for Basic Research. The work has been partially financially supported by the Russian State Research \# 0073-2018-0002, and by Government of Russian Federation, Grant 074-U01.

\section{References}

1. N. Hashimoto, K. Tomita, A. Kamimura, Y. Takinami, O. Matsumoto, International Internet of Things Summit, 157-162 (2014)

2. N. Sato, J. Kim, N. Hashimoto, K. Tomita, O. Matsumoto, A. Boyali, The First IEEE International Smart Cities Conference (2015)

3. J. Ashurst, B. Wagner, Western Journal of Emergency Medicine, 16 (5), 693 (2015)

4. K. Boniface, M. McKay, R. Lucas, A. Shaffer, N. Sikka, Annals of Emergency Medicine, 57 (4), 370374 (2011)

5. Department of Transportation. What is Distracted Driving. URL: http://www.distraction.gov/content/getthe-facts/ facts-and-statistics.html (2012)

6. National Center for Statistics and Analysis, Distracted Driving: 2014 Data, Traffic Safety Research Notes (2015)

7. Research Bulletin of National Solid Wastes Management Association, Distracted While Driving, https://wasterecycling.org/images/documents/resour ces/Research-Bulletin-Distracted-Driving.pdf

8. Traffic Safety Facts, Training Novice Drivers to Shorten Distraction Time, https://ntl.bts.gov/lib/61000/61600/61653/tt387.pdf

9. C. You et al, Proceedings of MobiSys'13, 13-26 (2013)

10. L. Bergasa, D. Almería, J. Almazán, J. Yebes, R. Arroyo, IEEE Intelligent Vehicles Symposium, 240-245 (2014)

11. T. Wang, G. Cardone, A. Corradi, L. Torresani, A. Campbell, Proceedings of the twelfth workshop on mobile computing systems \& application, 5 (2012)

12. M. Maslac, B. Antic, K. Lipovac, D. Pešic, N. Milutinovic, Transportation Research Part F, 52, 101-111 (2018)

13. Y. Zhao, T. Yamamoto, T. Morikawa, Traffic and Transportation Engineering (to be published)

14. F. Vicente, Z. Huang, X. Xiong, F. De La Torre, W. Zhang, D. Levi, IEEE Transactions on Intelligent Transportation Systems, 16 (4), 2014-2027 (2015)
15. N. Kamarud, A. Wahab A. Rahman, K. Ikhwan Mohamad Halim, International Conference on Informatics and Computing (ICIC), 186-191 (2016)

16. A. Smirnov, A. Kashevnik, I. Lashkov, N. Hashimoto, A. Boyali, Proceedings of the 17th conference of FRUCT Association, 201-209 (2015)

17. N. Hashimoto et al, 2016 19th Conference of Open Innovations Association (FRUCT) , 47-53 (2016)

18. Segway Japan, http://www.segway-japan.net. 